\title{
marges Marges
}

revue d'art contemporain Revue d'art contemporain

25 | 2017

Archives

\section{Éditorial}

Jérôme Glicenstein

\section{OpenEdition}

\author{
Journals
}

Édition électronique

URL : http://journals.openedition.org/marges/1306

DOI : ERREUR PDO dans /localdata/www-bin/Core/Core/Db/Db.class.php L.34 : SQLSTATE[HYO00]

[2006] MySQL server has gone away

ISSN : 2416-8742

\section{Éditeur}

Presses universitaires de Vincennes

\section{Édition imprimée}

Date de publication : 1 octobre 2017

Pagination : 5-7

ISBN : 978-2-84292-723-3

ISSN : $1767-7114$

\section{Référence électronique}

Jérôme Glicenstein, «Éditorial », Marges [En ligne], 25 | 2017, mis en ligne le 01 octobre 2017, consulté le 08 janvier 2021. URL : http://journals.openedition.org/marges/1306 ; DOI : https://doi.org/10.4000/ marges.1306 


\section{Éditorial}

Les archives jouent un rôle grandissant dans l'art contemporain, tant dans la pratique des artistes que dans celle des chercheurs. Le critique Hal Foster parle de "pulsion d'archive " [archival impulse], à propos d'artistes qui se servent d'archives comme d'un matériau pour leurs œuvres et de fait, de nombreux artistes mettent en œuvre un travail " archivistique " de rangement ou d'accumulation qui interroge les normes régissant les rapports sociaux aux objets du passé. Simultanément, l'évolution des pratiques artistiques rend les archives de plus en plus nécessaires: l'art contemporain, fréquemment conçu dans des formes éphémères, ne s'appréhende bien souvent que par ses traces et réminiscences (photographies, récits, enregistrements...). C'est de plus en plus grâce à la documentation conservée dans les archives d'artistes et d'institutions que l'étude de l'art devient possible. Cela pose alors la question de savoir qui constitue ces archives et dans quel cadre elles sont conservées. Toute opération d'archivage comporte en effet des sélections et des exclusions qui conditionnent les reconstructions historiques: celles-ci varient aussi à la suite des circonstances dans lesquelles les archives sont constituées et conservées. 
L'évolution récente du concept d'archive, de son statut et de ses différentes fonctions sociales invite à la réflexion. Loin d'être un dispositif neutre, la condition de l'archive, comme le note Derrida, est celle d'être un lieu d'autorité. L'archive peut être un instrument de pouvoir, imposant des récits idéologiques et des identités figées, mais elle peut aussi permettre de reconstruire à rebours l'histoire des « hommes infâmes » dont parle Foucault. Réfléchir au fonctionnement de l'archive permet ainsi de mettre en question non seulement les modes de fonctionnement, mais aussi la fragilité des institutions chargées de la conservation archivistique, révélant par là l'instabilité de la mémoire sociale.

Enfin, la réflexion critique sur le dispositif archivistique se fait d'autant plus nécessaire aujourd'hui qu'on assiste à une progressive fétichisation des archives: on les expose de plus en plus et leur prix monte sur le marché. Cette actuelle valorisation des archives peut sans doute être vue aussi comme un symptôme de leur disparition, au moins sous leur forme matérielle habituelle. Avec le développement de l'informatique, les archives personnelles disparaissent progressivement et les artistes comme les institutions se lancent dans un processus infini de numérisation des ressources disponibles. Les auteurs de ce numéro archivistes, artistes et théoriciens de l'art - réfléchissent tous à leur manière à ces questions.

Le premier texte, dû à Clothilde Roullier et Yann Potin des Archives nationales, permet de revenir sur l'histoire de la relation des artistes aux archives et à l'archivage, dans le contexte français. Les auteurs distinguent parmi les documents qui entourent les œuvres ceux qui sont produits par leurs auteurs et ceux qui émanent de l'administration, remarquant que des choix ont constamment été faits, qui orientent le regard des chercheurs - particulièrement depuis la création du Bureau des travaux d'art, puis du Ministère des affaires culturelles.

Le texte de Paul Bernard-Nouraud s'intéresse à l'usage que certains artistes font des documents d'archive. Les exemples d'Édouard Manet, Paul Klee, Gerhard Richter et quelques autres sont convoqués afin de montrer la plasticité de l'usage que font ces auteurs de documents tirés de l'actualité et versés au sein de « leur » archive. Maria Tyl prend, quant 
à elle, le parti inverse et s'attache davantage à l'étude de la construction collective d'une archive par des artistes. L'exemple qu'elle choisit, la galerie polonaise Foksal, lui permet de montrer que "l'archive vivante " peut être un ciment qui unit des artistes, voire un moyen de résister collectivement à un contexte défavorable à la création. Une situation similaire fait l'objet de l'enquête d'Elena Lespes-Munoz à propos des archives du Musée d'art contemporain de São Paulo (MAC-USP). Là aussi, en pleine dictature, les artistes et acteurs du musée inventent une forme de résistance qui passe par la production de documents en tout genre et notamment par la promotion de l'art postal et de formes documentaires pauvres. Les objets produits ne sont pas des œuvres au sens traditionnel et comme ils ne rentrent pas dans les catégories de la conservation, ils peuvent circuler plus librement. Les archives sont alors le lieu de la conservation de toutes ces activités: un lieu de liberté pour l'art.

Les quatre textes suivants partent de questions beaucoup plus spécifiques. Guillaume Sintès s'intéresse à la pratique de l'archive en danse, au travers du projet Waehner, et notamment aux problèmes posés par la recréation d'un projet chorégraphique déjà ancien. Les archives jouent évidemment un rôle central dans une telle configuration, permettant la reprise-transposition des œuvres dans le contexte contemporain.

La question posée par le texte de Nina Mansion est assez différente, bien que les problèmes rencontrés soient tout aussi importants. La question de l'archive de l'architecture contemporaine y est présentée comme complexe, puisqu'elle est « ce qui règle la production de discours en et sur l'histoire de l'architecture ». Une nouvelle fois, en un prolongement de la réflexion de Clothilde Roullier et Yann Potin, les archives produites par les architectes eux-mêmes s'opposent à leur mise en ordre par des institutions spécialisées.

Le texte de Roberta Agnese prolonge les mêmes questions, à partir du travail de trois photographes: d'un côté Larry Sultan et Mike Mandel - qui collectent et mettent en ordre des images trouvées comme des "preuves " de quelque chose qui n'apparaît pas - et de l'autre, Peter Piller, un artiste qui regroupe des images trouvées dans des catégories à première vue absurde. 
Le dernier texte du dossier, dû à Marlon Miguel, revient sur la pratique d'archivage de Fernand Deligny. Son célèbre projet s'accompagne en effet de toute une activité d'écriture, de documentation et de tournage de films, lesquels ont une destination qui n'est pas toujours explicite, mais permet d'asseoir le projet dans son ensemble.

Nous publions aussi deux entretiens sur le sujet. Le premier, réalisé avec le critique d'art américain Hal Foster, revient sur "The Archival Impulse », un article de 2004 qu'il avait consacré aux pratiques artistiques contemporaines faisant appel aux archives. Le second, qui réunit Don Foresta, Jean-Marie Dallet et Frédéric Curien, fait état de leur travail collectif autour des archives des pionniers de l'art vidéo Woody et Steina Vasulka (et de la manière dont ces archives sont réinvesties dans des démarches artistiques contemporaines).

Dans les varias de ce numéro nous publions un article de Marlène Lespes consacré aux pavillons marocains dans les Expositions coloniales en France avant la Seconde Guerre mondiale. Il est précédé d'un portfolio de Mabe Bethônico - lequel est pour la première fois dans l'histoire de Marges - en couleur.

Jérôme Glicenstein

Octobre 2017 